\title{
JuriGenT, un banco de datos jurídico neerlandés/español diferente
}

\section{JuriGenT, a different bilingual database of legal Dutch/Spanish}

\author{
Patricia VANDEN BULCKE y Carine DE GROOTE \\ Universiteit Gent \\ Patricia.VandenBulcke@ugent.be, Carine.DeGroote@ugent.be
}

Recibido: 29-9-2015. Revisado: 23-10-2016. Aceptado: 1-11-2016.

Resumen: JuriGenT es un banco de datos de terminología jurídica bilingüe neerlandés/ español, cuya publicación está prevista para septiembre de 2017. Desde la perspectiva de la traducción, JuriGenT se basa en la teoría del Skopos de Nord (1997), que implica una traducción "documental» (translation as a purposeful activity), y en el enfoque dirigido al destinatario (receiver-oriented approach) preconizado por Šarčević (2000). Ambas aproximaciones teóricas obligan a definir el papel del traductor, que será el de «representar» el texto fuente dentro del contexto jurídico fuente, si bien en otro idioma, para que el jurista pueda interpretarlo después en el contexto meta. En lo relativo a las teorías terminológicas, JuriGenT está basado en la teoría comunicativa de Cabré (1999) y la teoría sociocognitiva de Temmerman (2000). Ambas autoras insisten en la necesidad de definir al usuario del banco de datos, que en nuestro caso es el traductor y no el jurista. Este enfoque teórico nos ha llevado a tomar una serie de decisiones importantes con respecto a la selección de los términos y la conceptualización de la ficha terminográfica. JuriGenT ofrece una interfaz amigable que permite navegar rápidamente entre hiperónimos, hipónimos y términos coordinados en ambas lenguas.

Palabras clave: Terminología jurídica; neerlandés/español; asimetría conceptual; encaje textual.

Patricia VANDEN BULCKE y Carine DE GROOTE JuriGenT, un banco de datos jurídico neerlandés/ español diferente 


\begin{abstract}
JuriGenT is a bilingual database of legal Dutch/Spanish, which is being readied for publication in September 2017. As far as translation principles are concerned, JuriGenT relies on the Skopos translation theory of Nord (1997), which implies a «documentary» translation («translation as a purposeful activity»), and on the «receiver-oriented approach» promoted by Šarčević (2000). Both theories insist on the importance of defining the role of the translator, i.e. to «represent» the source text in the source context, but in another language, as the translation has to be interpreted by the legal expert in the target context. With respect to terminological principles, the approach relies on the communicative theory of Cabré (1999) and the sociocognitive approach of Temmerman (2000). Both authors insist on the need to define the user of the database: in our case the translator and not the lawyer. These theories have led to important decisions regarding the selection of the included terminology and the conceptualization of the terminological record. Finally, JuriGenT devotes attention to user-friendly consultation, allowing easy navigation to broader terms, narrower terms and related terms in both languages.
\end{abstract}

Keywords: Legal terminology; Dutch/Spanish; conceptual asymmetry; textual fit.

\title{
1. RELEVANCIA DE JURIGENT
}

Uno de los problemas que afrontan las lenguas de menor difusión, como el neerlandés, es la poca rentabilidad económica de sus diccionarios u otras herramientas lingüísticas, tanto generales como terminológicos, monolingües o bilingües (para una visión de conjunto véase Martin 2006, 55-59): el público meta ya es escaso de por sí y se restringe aún más en el caso de la terminología especializada. Más aún es así en el campo de la lengua jurídica, que viene determinada no solo por el idioma, sino también por el sistema que trata. En el caso del neerlandés eso supone restringirse, o bien al público meta neerlandés, o bien al público meta belga.

El panorama de las herramientas bilingües neerlandés/español de terminología jurídica es muy limitado. En papel, de neerlandés a español, solo existe el DIC Oosterveld (1990), que pretende abarcar tanto la terminología belga como la neerlandesa, aunque cabe subrayar que el enfoque está sobre todo en el derecho neerlandés; en la nueva versión electrónica que se está preparando, la terminología belga incluso quedará excluida. En dirección opuesta existe el DIC Boutmans (2005), que pone el acento en la terminología administrativa (España y Latinoamérica), pero al tratarse de una publicación propia, resulta menos accesible. Es cierto que también existen herramientas electrónicas, es decir, bancos de datos terminológicos o de textos europeos, como IATE y EUR-Lex, que recogen la terminología jurídica en ambos idiomas tratados. Sin embargo, el usuario tiene que ser consciente de que recogen el llamado «eurolecto", cuyas particularidades se pueden describir como sigue: a) el sistema jurídico subyacente es el europeo y, por consiguiente, uniforme, b) el neerlandés se usa como lengua

Patricia VANDEN BULCKE y Carine DE GROOTE JuriGenT, un banco de datos jurídico neerlandés/ español diferente 
genérica, sin etiquetado de neerlandés de Bélgica o de los Países Bajos, y c) la terminología procede de traducciones de textos comunitarios y no queda claro si el término en cuestión es el término original o una traducción. Cabe decir que últimamente se hacen esfuerzos para señalar en EUR-Lex cuál ha sido el texto fuente. Además, se han iniciado proyectos como Eurolect Observatory cuyo objetivo es, entre otros, estudiar las diferencias terminológicas entre el neerlandés de Bélgica y el de los Países Bajos a la hora de transponer las leyes comunitarias en nacionales.

Con JuriGenT hemos querido diseñar un banco de datos jurídico bilingüe que recoja terminología «nacional», es decir, usada en Bélgica y en España. Pero JuriGenT obedece a más criterios, la mayoría de ellos originados por los nuevos modelos teóricos de traducción y terminología de las últimas décadas. Se describen a continuación.

\section{MARCO TEÓRICO DE JURIGENT}

Diseñar un banco de datos terminológico implica, según Temmerman (2000, 235), Cabréetal. (2003) y Cabré (2005), reflexionar sobre el usuario del mismo y sus necesidades. Al definir al usuario de JuriGenT como el traductor jurídico, se llega automáticamente a la pregunta de la especificidad de la traducción jurídica. Nord (1997), en su teoría del Skopos, considera que traducir es una actividad que se efectúa en función de un propósito determinado (translation as a purposeful activity), y en este sentido distingue entre la traducción documental y la traducción instrumental. A continuación citamos la definición de la primera, la documental, ya que se aplica directamente a lo que, en nuestra opinión, debe ser una traducción jurídica: informar sobre la situación jurídica expresada en el documento fuente, sin «localizarla» en una situación jurídica meta.

$[\ldots]$ the documentary translation type, is first and foremost a metatext, being a targetculture text informing about a source-culture text and any of its aspects and dimensions [...] and as such, its communicative function will be realized in an indirect way: e.g., informing target-culture addressees about a source-culture author referring to an object of the world (from a source-culture point of view) [...]. (Nord 1997, 49)

La descripción de Engberg (2013) de lo que es la traducción jurídica se asemeja a la definición general de la traducción documental de Nord:

If we start by looking at the description of the act of translation, we observe that it concentrates upon producing texts that convey the same choice of knowledge elements in a target text as those presented in a source text. Thus, the task is, so to speak of depicting a previous instance of communication. What a lawyer does is different: the lawyer solves social problems on the basis of legal knowledge. (Engberg 2013, 30)

Patricia VANDEN BULCKE y Carine DE GROOTE JuriGenT, un banco de datos jurídico neerlandés/ español diferente 
Resulta interesante su observación sobre las distintas tareas que llevan a cabo el traductor y el abogado (o jurista): el primero describe, el segundo interpreta. Eso nos ha llevado a especificar al usuario de JuriGenT no solo como «traductor», sino también como «no jurista» (lo que no implica que no pueda serlo).

Šarčević (1997) ya abordó esta especificidad de la traducción jurídica añadiendo una dimensión: the uniform intent, con lo que quiere decir que el documento jurídico traducido debe expresar lo que el jurista redactor del texto fuente quiso decir:

The translator's first consideration is no longer fidelity to the source text but rather fidelity to the uniform intent of the single instrument, i.e. what the legislator or negotiators intended to say. (Šarčević 1997, 112)

DeahíqueŠarčević $(1997,112)$ hable del «traductor emancipado». Cabe observar que, en la práctica, el traductor incluso puede verse obligado a interpretar o a tomar posición, ya sea a causa de las asimetrías lingüísticas (palabras/términos que forzosamente deben traducirse por una palabra/un término más concreta/o y que casi no dejan parafrasearse), o a causa de las asimetrías conceptuales entre los sistemas jurídicos tratados. Como ilustración del primer caso queremos señalar el verbo 'poder', que en neerlandés se traduce o bien por 'kunnen', o bien por 'mogen' (Vanden Bulcke 2013, 29-30), y el sustantivo 'responsabilidad', que se traduce o bien por 'verantwoordelijkheid' (concepto moral), o bien por 'aansprakelijkheid' (concepto jurídico). Como ilustración del segundo caso señalamos el término 'sentencia', que se traduce por 'vonnis' (sentencia de un juzgado) o por 'arrest' (sentencia de un tribunal). Ante este problema hemos optado en JuriGenT por no presentar soluciones prefabricadas, sino por ofrecer información que ayude a seleccionar el equivalente idóneo en el otro idioma.

Y, por último, JuriGenT bebe de la teoría comunicativa de la terminología de Cabré (1999) y de la teoría sociocognitiva de la terminología de Temmerman (2000). Estas teorías subrayan que los términos, igual que las palabras de la lengua general, "se actualizan", es decir, que se usan siempre dentro de un contexto determinado, en una situación discursiva determinada, como unidades determinadas cognitivamente. Las unidades terminológicas «por lo tanto están muy lejos de ser el binomio conceptodesignación de límites precisos y valor universal que durante mucho tiempo postuló la teoría terminológica» (Cabré et al. 2003, 2). De ahí la decisión de tratar en JuriGenT una nomenclatura ligada a un tipo discursivo o género específico, en este caso los estatutos societarios, y de trabajar con un corpus en ambos idiomas con el fin de poder describir el comportamiento específico de los términos dentro de su contexto.

Todas las influencias descritas arriba han dado lugar a una serie de premisas que se describen a continuación. Cada una de ellas entraña consecuencias prácticas para el diseño y la elaboración del banco de datos, algunas de las cuales describiremos en el apartado 4.

Patricia VANDEN BULCKE y Carine DE GROOTE JuriGenT, un banco de datos jurídico neerlandés/ español diferente 


\section{PREMISAS DE JURIGENT}

Las premisas de JuriGenT son las siguientes: es un banco de datos

1. elaborado en MultiTerm, un programa de gestión terminológica bastante difundido;

2. cuyas unidades proceden de dos corpus comparables, uno en neerlandés y otro en español, de 200000 palabras cada uno;

3. que recoge terminología del derecho de sociedades, más específicamente del género textual de los estatutos;

4. que trata la terminología ligada a dos sistemas jurídicos, el belga y el español; se dirige, sin embargo, al usuario neerlandófono, lo que implica que la lengua vernácula de las fichas es el neerlandés;

5. que es útil para la traducción del neerlandés al español y al revés, con un diseño que permite tratar las asimetrías lingüísticas y conceptuales (véase 7);

6. que se consulta alfabéticamente, pero que ofrece al mismo tiempo una estructuración conceptual, ya que las fichas recogen los hiperónimos, hipónimos y términos coordinados;

7. cuyas fichas están diseñadas en función de un enfoque onomasiológico, es decir, con una descripción genérica del concepto, aplicable tanto al concepto belga como al concepto español, pero con la posibilidad de anotar en un campo aparte (el llamado «campo de equivalencia»), por idioma, las asimetrías, las discrepancias o la ausencia de equivalencia respecto al concepto descrito;

8. que se dirige a un público meta específico, a saber, al traductor y no al jurista (Temmerman 2000, 235; Cabré et al. 2003; Cabré 2005)

JuriGenT contiene unas 1300 entradas, a las que corresponden unas 6000 unidades elaboradas.

\section{CONSECUENCIAS DE LAS PREMISAS ADOPTADAS E ILUSTRACIONES}

Las premisas arriba citadas tienen una influencia directa sobre el diseño de la ficha terminológica. Se pueden agrupar en función de las consecuencias que conllevan: a) las premisas 3 y 4 , e indirectamente también la 5 y la 7 , tienen que ver con la especificidad de la terminología jurídica y requerirán una solución conjunta (cf. 4.1), b) la premisa 2 tiene que ver con la contextualización de los términos, que también requerirá especial atención en las fichas (cf. 4.2), c) la premisa 6 es más bien de índole práctica y su realización solo es posible gracias a la premisa 1, es decir, el uso de una aplicación

Patricia VANDEN BULCKE y Carine DE GROOTE JuriGenT, un banco de datos jurídico neerlandés/ español diferente 
informática y, d) la premisa 8, la más específica de JuriGenT, afecta especialmente a la manera de abordar la descripción conceptual (4.3). Aun así la principal consecuencia de la premisa 8 concierne a la selección de las unidades que poblarán el banco de datos (4.4).

Antes de desarrollar este apartado es oportuno presentar brevemente la estructura general de la ficha terminológica usada. La ficha se divide en 4 partes: la primera, que se podría denominar «hoja de traducción» contiene metadatos, como el número de la entrada, el campo tratado, el proyecto..., una descripción del concepto (enfoque onomasiológico) y todos los equivalentes en ambos idiomas, es decir, término preferente y sinónimos, para que el usuario tenga desde el principio una visión de conjunto (véanse las figuras 1, 2, 5 y 6); la segunda y tercera parte se llaman respectivamente «hoja de lengua» $y$ «hoja de texto" y acompañan a cada uno de los términos recogidos en la «hoja de traducción»: la primera describe datos lingüísticos (véanse las figuras 3 , 4 y parte de $7 a$ y $7 b$ ), en tanto que la segunda recoge una definición extraída de un diccionario jurídico así como contextos de uso (véase parte de las figuras 7a y 7b).

\subsection{Campo específico para anotar equivalencias asimétricas}

Es comúnmente aceptado que la nomenclatura jurídica está ligada, antes que a un idioma, a un sistema jurídico y que estos difieren tanto entre países como entre regiones de un mismo país. Véanse al respecto Kisch (1973), De Groot (1987, 1988, 1996), Gémar (1988, 2002), Šarčević (1991, 1997), Thiry (1997, 2000, 2009), Mayoral (2002, 2003), Vanden Bulcke (1993, 1995, 1997, 2009, 2011), Vanden Bulcke y Héroguel (2011), entre otros. De ahí que a la premisa 3 (especialización temática: derecho de sociedades) hayamos añadido la premisa 4: la nomenclatura de dos sistemas específicos, el belga y el español. Para JuriGenT, esto significa concretamente que entre los dos sistemas tratados puede haber falta de simetría (conceptos inexistentes en uno de los dos sistemas) o puede haber asimetría (conceptos coincidentes solo parcialmente con el otro sistema).

En realidad, se dan muy pocos casos de falta de simetría, es decir, de conceptos (instituciones $\mathrm{u}$ otros) totalmente inexistentes en uno de los dos sistemas. Bélgica tiene, por ejemplo, el 'Kruispuntbank van Ondernemingen' y España la 'Junta Universal', pero en la mayoría de los casos existe en el otro sistema un concepto parecido. Merece especial atención el caso de juzgados y tribunales, tipos de sociedades, leyes, entidades... El uso de los términos pertenecientes a estas categorías oscila entre genérico y nombre propio, y tienen muy a menudo un equivalente funcional en el otro sistema. Véanse, por ejemplo, 'Belgisch Staatsblad'/'Boletín Oficial del Estado', 'Nationale Bank van België'/'Banco de España', 'Commissie voor het Bank-, Financieen Assurantiewezen'/‘Comisión Nacional del Mercado de Valores', etcétera. Dado

Patricia VANDEN BULCKE y Carine DE GROOTE JuriGenT, un banco de datos jurídico neerlandés/ español diferente vol. 2-2, December 2016, 15-38

elSSN: 2444-1961

(C) Ediciones Universidad de Salamanca - CC BY-NC-ND 
que la localización no tiene cabida en la traducción jurídica (cf. apartado 2), tratamos estos casos como conceptos exclusivos de un sistema y añadimos la etiqueta «Bélgica»/«España». Como término equivalente en el otro idioma proponemos, o bien un neologismo marcado con asterisco para que el usuario sepa que el término propuesto no existe en el otro sistema, o bien, de encontrarse una traducción en las fuentes secundarias DIC Oosterveld, EUR-Lex o IATE, la traducción encontrada, con referencia a las fuentes. Ya que en estos casos puede ser útil tener más información sobre el posible equivalente en el otro sistema, sobre el porqué del neologismo o sobre alguna precisión diacrónica, se ha introducido en el diseño de la ficha un campo llamado «de equivalencia». En Vanden Bulcke $(2011,25-27)$ se ilustra el uso de este campo en las figuras 5, 6 y 7, que representan respectivamente las fichas de 'Wetboek van Vennootschappen'/‘Código de Sociedades'^, 'coöperatieve vennootschap met onbeperkte aansprakelijkheid'/'sociedad cooperativa de responsabilidad ilimitada'^ y 'Commissie voor het Bank-, Financie- en Assurantiewezen'/'Comisión controladora de la Banca y del Sector financiero y de Seguros'*. A continuación ilustramos el uso de un concepto inexistente en uno de los sistemas con la ficha de 'procurador'/'procesv ertegenwoordiger'.

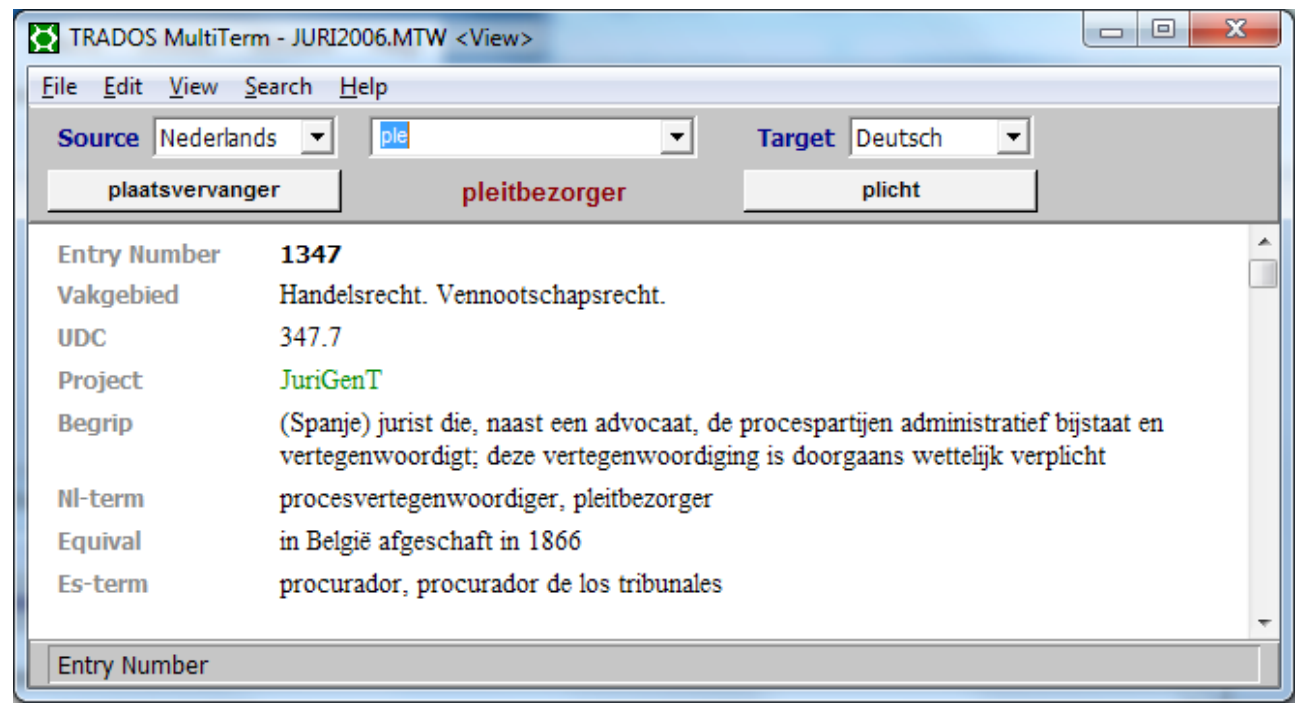

Figura 1. JuriGenT: 'procurador'/'procesvertegenwoordiger' (hoja de traducción)

Los casos de asimetría conceptual entre términos de distintos sistemas son más frecuentes. El campo "de equivalencia» sirve para anotar las divergencias. Hay casos de una equivalencia equilibrada (1 término/1 término), como 'notaris'/'notario', 'met de zorgvuldigheid van een goed koopman'/'con la diligencia de un ordenado empresario

Patricia VANDEN BULCKE y Carine DE GROOTE JuriGenT, un banco de datos jurídico neerlandés/ español diferente 
y representante leal', pero donde cabe precisar que uno de ambos conceptos es más amplio. También hay casos de una equivalencia desequilibrada (1 término/2 términos) como 'akten verlijden' frente a 'autorizar escrituras' u 'otorgar escrituras', 'sentencia' frente a 'vonnis' o 'arrest', 'aandeel' frente a 'acción' o 'participación', etcétera, donde el campo de equivalencia sirve para señalar la particularidad de la doble terminología de un sistema frente a la unicidad terminológica del otro. Lo ilustramos a continuación mediante la ficha de 'aandeel'/'acción' o 'participación'. De la información en el campo de equivalencia se puede deducir que cuando la palabra 'aandeel' hace referencia a una 'nv', se traduce por 'acción', y que cuando remite a una 'bvba', se traduce por 'participación'; al revés, tanto 'acción' como 'participación' se traducen por 'aandeel'. Finalmente, hay casos de equivalencia muy compleja como 'bestuurder' o 'zaakvoerder' frente a 'consejero' o 'administrador', donde hay asimetría entre los términos tanto a nivel de cada sistema (en neerlandés, el uso de los términos viene dictado por el tipo de sociedad; y en español, por el número de «personas» y por el hecho de que el referente pertenezca o no a un consejo de administración) como a nivel intersistémico (los criterios discriminatorios son distintos en ambos sistemas y por eso no hay equivalencias claras). En este caso se apuntan las asimetrías por idioma y se formula un concepto "contenedor» que sirve de referencia (véase Vanden Bulcke 2009).

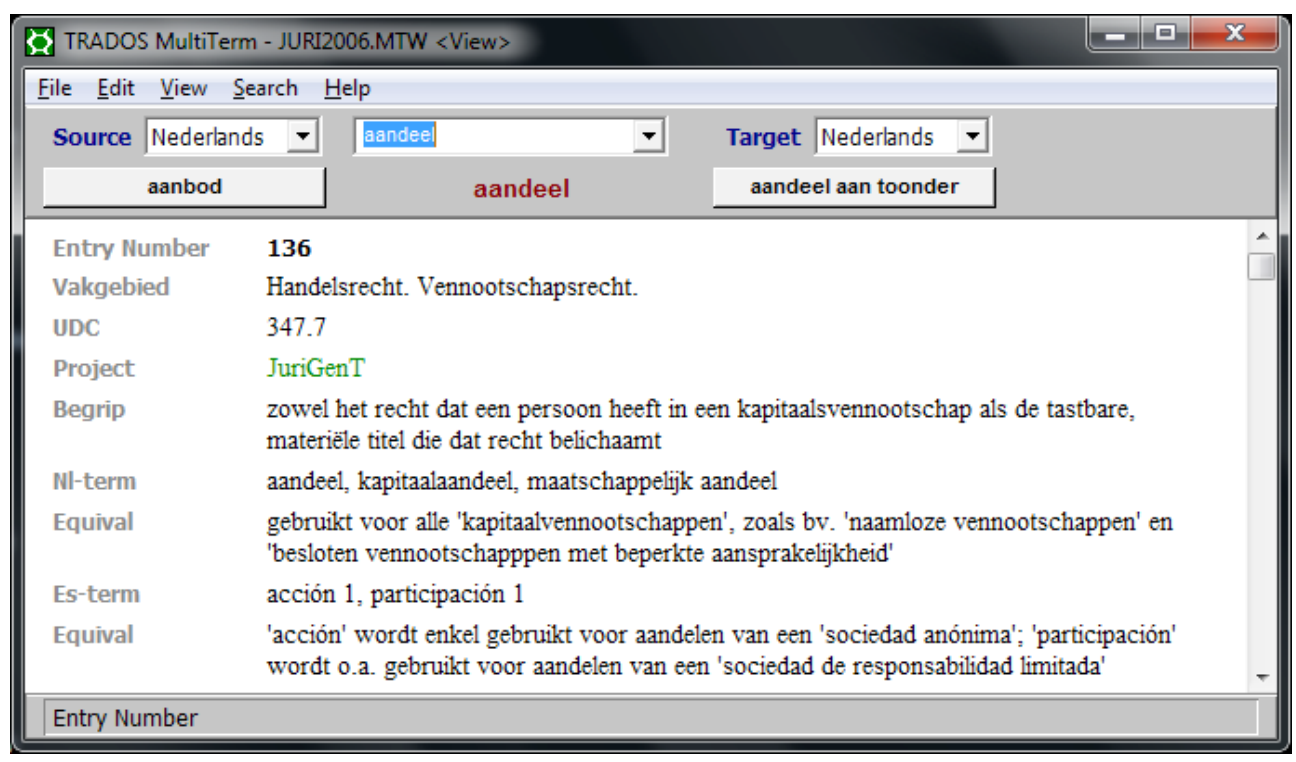

Figura 2. JuriGenT: 'aandeel'/‘acción' o 'participación' (hoja de traducción)

Patricia VANDEN BULCKE y Carine DE GROOTE JuriGenT, un banco de datos jurídico neerlandés/ español diferente 


\subsection{Campos específicos para colocaciones y contextos}

Al extraer las unidades de JuriGenT de un corpus, se garantiza que estas aparezcan en contexto. De la observación de los términos en su contexto se puede deducir una gran cantidad de información de índole diversa, no únicamente semántica: sintácticadistribucional (combinaciones de las unidades con paradigmas de categorías de palabras), gramatical (uso de los tiempos, cf. Vanden Bulcke 2013), fraseológica (expresiones discursivas frecuentes con un grado variable de fijación), semánticosintáctica (maneras de expresar contenidos especializados, por ejemplo mediante sujeto animado, sujeto inanimado), pragmática (manera de hacer referencias legales), etcétera. Toda esta información contribuye a una traducción natural y fluida, lo que en los estudios sobre traducción se ha acuñado como textual fit, "encaje o adecuación textual» (Chesterman 2004; Biel 2014). Como el banco de datos se dirige al traductor, y habida cuenta de que un «encaje textual adecuado» aumenta la aceptabilidad y el potencial comunicativo del texto traducido, además de facilitar su comprensión (Biel 2014), nos ha parecido imprescindible dar cabida a esta información en las fichas.

El banco de datos presenta, por consiguiente, fichas que contienen a) un campo específico para «colocaciones» y b) un campo específico para contextos. Manejamos una definición bastante amplia de "colocación» (véase Vanden Bulcke y De Groote 2011), basándonos en la definición que ofrece Lorente (2001, 21-22) para «fraseología especializada»: a) unidad de conocimiento en un contexto especializado, b) sintagma u oración, c) no lexicalizado totalmente y d) que contiene una unidad terminológica (UT) como mínimo. Implica tanto la combinatoria de las UT con categorías de palabras resultantes del método KWIC, key words in context (uso de un programa de concordancia), por ejemplo V + UT, Adj + UT, Nombre + determinante + UT..., como fraseología de diverso grado de lexicalización. En la medida de lo posible, intentamos relacionar las colocaciones del español con las del neerlandés, y viceversa: véase la Tabla 1 abajo, donde enumeramos las "colocaciones» de la UT 'acta'/'notulen' y sinónimos. Pero como las unidades proceden de dos corpus comparables y no paralelos, no hay equivalencia en todos los casos.

En las colocaciones, los sinónimos no son automáticamente intercambiables, lo cual subraya la importancia del término «en su contexto de uso» (lo que GoźdźRoszokowski $(2013,102)$ llama collocational behaviour). Así, en el ejemplo abajo citado, las colocaciones mencionadas le ayudan al traductor del neerlandés al español a ver que 'emitir' no se usa con 'acta' y sí con 'informe', y, que 'bijgevoegd' se puede traducir por un verbo, 'acompañarse de', o por un adjetivo, 'correspondiente'.

Patricia VANDEN BULCKE y Carine DE GROOTE JuriGenT, un banco de datos jurídico neerlandés/ español diferente 


\begin{tabular}{|l|l|}
\hline \multicolumn{1}{|c|}{ Colocaciones de 'acta' } & Colocaciones de 'informe' \\
\hline $\begin{array}{l}\text { la propia acta; } \\
\text { acta correspondiente; } \\
\text { (aprobar/certificar/extender/levantar/redactar/transcribir) } \\
\text { actas; }\end{array}$ & $\begin{array}{l}\text { redactar informes; } \\
\text { emitir informe sobre }\end{array}$ \\
$\begin{array}{l}\text { consignar/hacer constar en el acta; } \\
\text { el acta se acompañará de; } \\
\text { aprobar actas en cualquiera de las formas (admitidas en } \\
\text { derecho/previstas en la ley/que regula la ley); } \\
\text { acta extendida o transcrita en un libro registro especial }\end{array}$ \\
\hline
\end{tabular}

\begin{tabular}{|c|c|c|}
\hline Colocaciones de 'notulen' & $\begin{array}{l}\text { Colocaciones de } \\
\text { 'proces-verbaal' }\end{array}$ & Colocaciones de 'verslag' \\
\hline $\begin{array}{l}\text { voornoemde notulen; } \\
\text { notulen opmaken/opstellen; } \\
\text { in de notulen opnemen/ } \\
\text { optekenen/ vastleggen; } \\
\text { de notulen worden in een } \\
\text { speciaal register ((bij)gehouden/ } \\
\text { ingeschreven/ingelast/op- } \\
\text { genomen/ingebonden) }\end{array}$ & $\begin{array}{l}\text { processen-verbaal } \\
\text { opmaken/opstellen; } \\
\text { in een proces-verbaal } \\
\text { opnemen; } \\
\text { ingevolge proces-verbaal } \\
\text { van }\end{array}$ & $\begin{array}{l}\text { (bijgevoegd/bijzonder/ } \\
\text { jaarlijks/periodiek/ } \\
\text { semestrieel) verslag; } \\
\text { verslagen opstellen; } \\
\text { in het verslag opnemen; } \\
\text { optekenen in de vorm van } \\
\text { een verslag }\end{array}$ \\
\hline
\end{tabular}

Tabla 1. Colocaciones de 'acta'/'notulen' y sinónimos

La mayor dificultad derivada del uso de corpus comparables - en el caso de JuriGenT además bastante reducidos - es que ni se dan todas las posibilidades, ni se da para cada colocación un equivalente en el otro idioma. Necesariamente hay que recurrir a las competencias lingüísticas y traductológicas del potencial usuario del banco de datos, el traductor. JuriGenT apela a cierta emancipación de su usuario, cierta autonomía en la toma de decisiones. La colocación neerlandesa 'BTW-plichtig zijn', que figura en la ficha de 'belasting over de toegevoegde waarde'/‘impuesto sobre el valor añadido', tiene como colocaciones equivalentes en español 'ser sujeto pasivo del IVA', 'estar sometido al IVA' y 'estar obligado a pagar el IVA'. Al traductor le corresponde sopesar los matices.

Patricia VANDEN BULCKE y Carine DE GROOTE JuriGenT, un banco de datos jurídico neerlandés/ español diferente 
Para las colocaciones interesantes desde un punto de vista contrastivo - por ejemplo porque apuntan a estructuras semántico-sintácticas discrepantes- se elabora una ficha. Ejemplo: 'verwezenlijking van het doel van de vennootschap', 'verwezenlijking van het maatschappelijk doel' frente a 'ejercicio de las actividades comprendidas en el objeto social', 'desarrollo de las actividades propias del objeto social'. Aquí la discrepancia se sitúa en la profusión de palabras ('comprendidas en', 'propias de'). En las publicaciones anteriores sobre JuriGenT arriba citadas se recogen más ejemplos.

\subsection{Descripción conceptual para el traductor, con exclusión del referente jurídico}

La premisa 7 define el enfoque de JuriGenT como onomasiológico, lo que a primera vista puede parecer contradictorio en terminografía jurídica, ya que esta debe trasponer conceptos íntimamente ligados a un sistema. Véase a este respecto la clara posición de DIC Oosterveld, como diccionario unidireccional de neerlandés a español, con un índice alfabético español-neerlandés. Sin embargo, como ya se ha mencionado en 4.1, esta deficiencia se suple en JuriGenT mediante el "campo de equivalencia", que permite anotar equivalencias asimétricas. La premisa 6 , por su parte, incluye una estructuración conceptual del dominio tratado, con vinculaciones a base de relaciones lógicas y ontológicas sobre todo. JuriGenT señala en las fichas términos coordinados, hiperónimos e hipónimos, lo que da lugar a descripciones conceptuales estructuradas. Para que haya correspondencia entre el neerlandés y el español, se parte para la presentación de estos términos del neerlandés, en orden alfabético. Este orden se mantiene en español. Véanse a título ilustrativo las figuras 3 y 4, con los hipónimos de 'document'/'documento', entre otros campos

Patricia VANDEN BULCKE y Carine DE GROOTE JuriGenT, un banco de datos jurídico neerlandés/ español diferente 


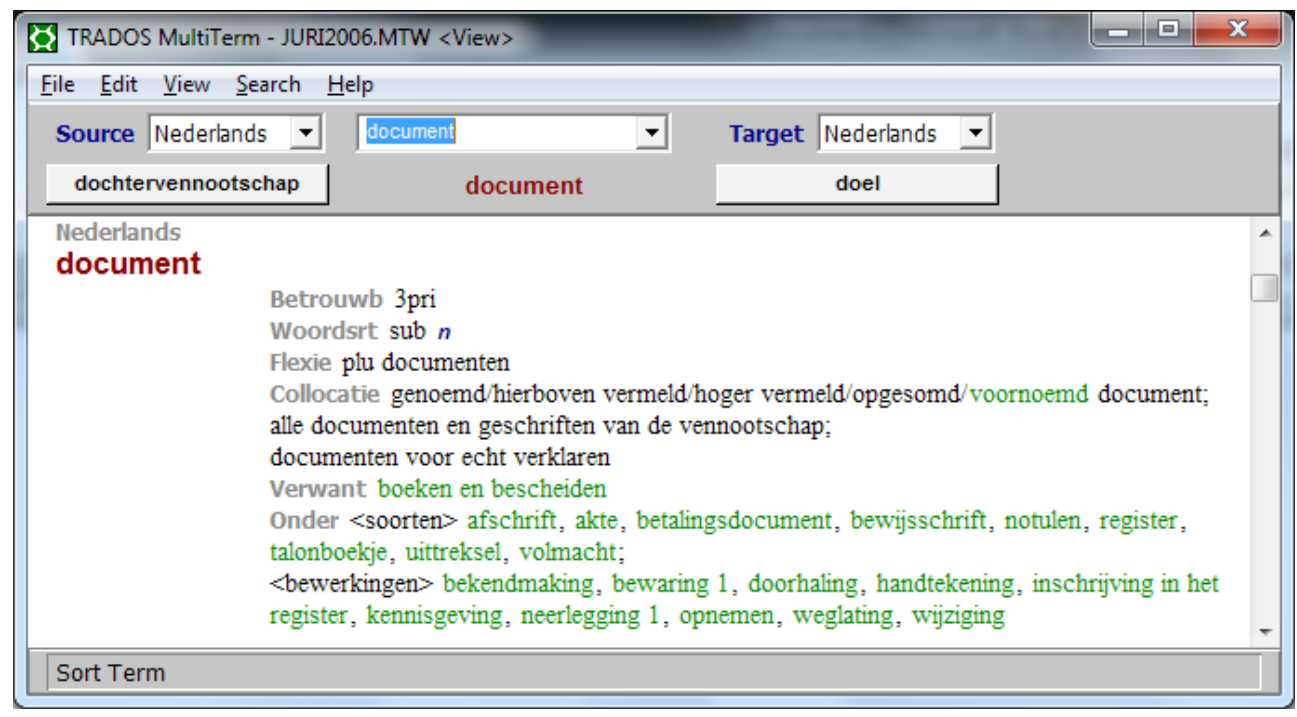

Figura 3. JuriGenT: 'document' (hoja de lengua)

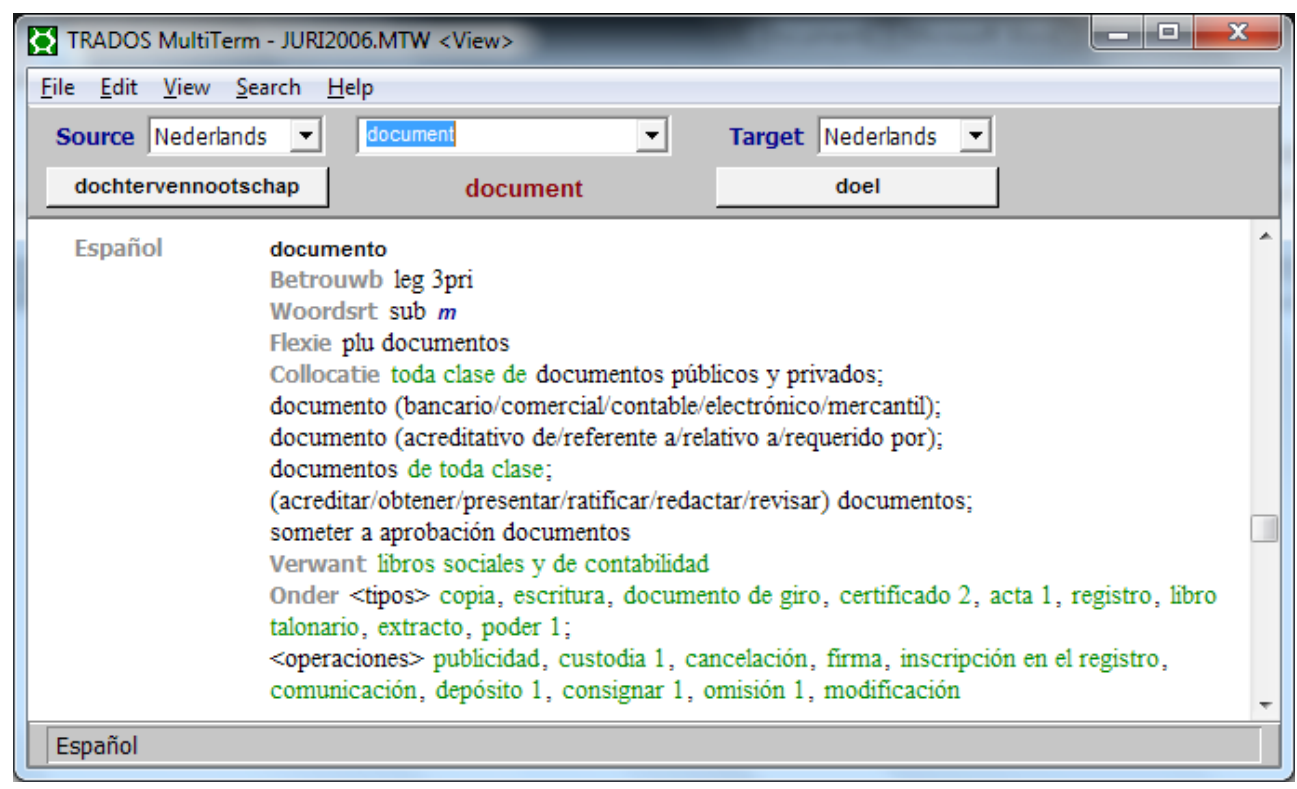

Figura 4. JuriGenT: ‘documento’' (hoja de lengua)

La descripción conceptual de cada hipónimo de la serie «tipos de» empieza con «documento que», seguido por la especificidad del hipónimo. Son descripciones que

Patricia VANDEN BULCKE y Carine DE GROOTE JuriGenT, un banco de datos jurídico neerlandés/ español diferente
CLINA

vol. 2-2, December 2016, 15-38

eISSN: 2444-1961

(C) Ediciones Universidad de Salamanca - CC BY-NC-ND 
satisfacen al traductor porque delimitan en grandes líneas los conceptos, pero que pueden no satisfacer al jurista por la falta de matices o referentes jurídicos. Ahora bien, como la tarea del traductor se limita a "describir», y no consiste en «interpretar» (cf. 2), este no necesita sistemáticamente la información jurídica que le proporciona el diccionario jurídico. Muy a menudo esa información le dificulta la comprensión del concepto. En la definición «jurídica» de 'escritura fundacional' más abajo se menciona, por ejemplo, que esta implica la celebración de una junta general en la que hay que nombrar administradores, y que tiene que inscribirse en el registro mercantil para tener validez, información que el traductor no necesita saber. JuriGenT también omite en la descripción conceptual cualquier referencia a la legislación. Sin embargo, si encontramos una definición en un diccionario jurídico la añadimos a las fichas, aunque cabe señalar que el banco de datos contiene una gran cantidad de términos que no figuran en los diccionarios y cuya ficha, por lo tanto, carece de definición «jurídica». A continuación citamos una descripción conceptual y una definición jurídica del término 'documento' y del término 'escritura fundacional'.

\section{documento (concepto):}

geschreven of gedrukt (bewijs)stuk [pieza (de prueba) escrita o impresa]

\section{documento (definición):}

véase 'patrimonio documental': conocido también como patrimonio documental y bibliográfico, es la parte del patrimonio histórico constituida por cuantos bienes, reunidos o no en archivos y bibliotecas, sean declarados de tal condición. A estos efectos se entiende por documento toda expresión en lenguaje natural o convencional y cualquier otra expresión gráfica, sonora o en imagen, recogida en cualquier tipo de soporte material, incluso los informáticos, quedando excluidos los ejemplares no originales de ediciones; [...] (^DIC Ribó Durán 2005^)

\section{escritura fundacional (concepto)}

authentieke akte, voor notaris verleden, waarbij een vennootschap wordt opgericht en waarin o.a. de statuten worden vastgelegd [escritura pública, autorizada por notario, en la que se funda una sociedad y en la que constan los estatutos sociales]

\section{escritura fundacional (definición)}

véase 'convenio de sociedad anónima': contrato societario que origina la sociedad anónima. Debe formalizarse en escritura pública. Se hará constar el acuerdo fundacional juntamente con los estatutos sociales. La suma de ambas partes forma la escritura de fundación o escritura de constitución de la sociedad anónima, que suele incluir la celebración de una junta general en la que se nombran el o los administradores de la compañía. Hasta que dicha escritura se inscriba en el registro mercantil, no hay sociedad anónima. [...] (^DIC Ribó Durán 2005^)

Patricia VANDEN BULCKE y Carine DE GROOTE JuriGenT, un banco de datos jurídico neerlandés/ español diferente 
En esta tarea de delimitación conceptual hemos tenido que afrontar el problema de la sinonimia que, si ya es abundante en textos legales en general, lo es aún más en los estatutos.

Translators dealing with legal language inevitably face a bewildering range of synonymous or near-synonymous terms or words appearing in virtually all legal texts. (Goźdź-Roszokowski 2013, 94)

En el género de los estatutos, las listas de términos semánticamente interrelacionados constituyen un rasgo estilístico determinante. Ello se debe a que los estatutos, que rigen «la organización y funcionamiento de la compañía mercantil» (DIC Ribó Durán 2005), tienen que combinar la precisión jurídica con la vaguedad propia de una actitud flexible en cuantas situaciones se presentan o presenten. Algo parecido describe Bhatia $(1994,144$, citado en Andrades 2013, 405) en relación con los binomios: «an extremely effective linguistic device to make the legal document precise as well as all-inclusive». En los estatutos se trata tanto de binomios ('gobierno y administración'; 'límites y requisitos'; 'actos y negocios jurídicos'; 'bons en obligaties') como de trinomios ('creación, supresión o traslado'; 'rige, gobierna y administra'; 'actos, contratos o negocios jurídicos'; 'schulden, lasten en kosten') o multinomios (véase el ejemplo abajo citado), las llamadas «cadenas». La relación semántica de los componentes va de la sinonimia o casi-sinonimia ('rige, gobierna y administra'; 'sucursales, agencias, delegaciones, oficinas y representaciones'; 'bons en obligaties') a la afinidad que puede llegar a manifestarse bajo múltiples variaciones ('creación, supresión o traslado'; 'disolución, fusión, escisión y transformación'; 'schulden, lasten en kosten'). La falta de fijación induce a creer que se crean ad hoc (Chromà 2001, citado en Goźdź-Roszokowski 2013). A continuación un ejemplo de un «binomio» y sus variaciones hasta formar una "cadena», en español y en neerlandés:

- sucursales y agencias

- sucursales, agencias o delegaciones

- centrales, subcentrales, sucursales, agencias o despachos

- sucursales, agencias, delegaciones, oficinas y representaciones

- bijkantoren en agentschappen

- bijhuizen, kantoren en agentschappen

- administratiekantoren, bedrijfszetels, succursales en agentschappen

- bijkantoren, dochterbedrijven, agentschappen, depots en representatiekantoren

- bijkantoren, bedrijfszetels, filialen, dochtermaatschappijen, agentschappen, depots en vertegenwoordigingen

Patricia VANDEN BULCKE y Carine DE GROOTE JuriGenT, un banco de datos jurídico neerlandés/ español diferente 
En cuanto a la traducción de estos binomios, trinomios y multinomios, GoźdźRoszokowski (2013) formula dos observaciones interesantes:

Existing specialized lexicographical resources are usually of limited use as they do not effectively clarify the nuances of meaning and usage involved in such terms. (GoźdźRoszokowski 2013, 95)

He or she (the translator) must determine the degree of fixedness and idiomaticity of synonymous chains and, ultimately, determine whether such lexical items serve any function. (Goźdź-Roszokowski 2013, 97)

Primero cabe señalar, como se verá en adelante, que la primera cita pone de manifiesto una laguna real, y que suplirla es tarea ardua, por no decir imposible: el uso de estos términos en forma de «listas» no ofrece una contextualización útil, y las fuentes jurídicas no se ocupan de delimitar unos términos que a primera vista parecen "Comunes». En cualquier caso, hemos adoptado el punto de vista de la segunda cita, ya que JuriGenT se dirige al traductor: si hay muestras de fijación e idiomaticidad, los binomios, trinomios, multinomios o cadenas se recogen, en ambos idiomas, como colocaciones o parte de colocaciones. Es preciso recalcar que el fenómeno es más profuso en español que en neerlandés. Si la fijación es menor intentamos establecer una distinción entre los conceptos -en un intento por abarcar cuantas variantes sean posibles - y los tratamos bajo los términos correspondientes, con resultados no siempre satisfactorios. En la figura 5, por ejemplo, intentamos desambiguar los conceptos de 'sede administrativa' y 'domicilio social', remitiendo en la descripción conceptual del primer término al segundo término y señalando que puede ocurrir que el referente de ambos términos coincida. Al mismo tiempo, tratamos de aclarar el uso específico del término español 'oficina de la administración de la sociedad', señalando que este solo se usa cuando su referente no coincide con el domicilio social.

Patricia VANDEN BULCKE y Carine DE GROOTE JuriGenT, un banco de datos jurídico neerlandés/ español diferente 


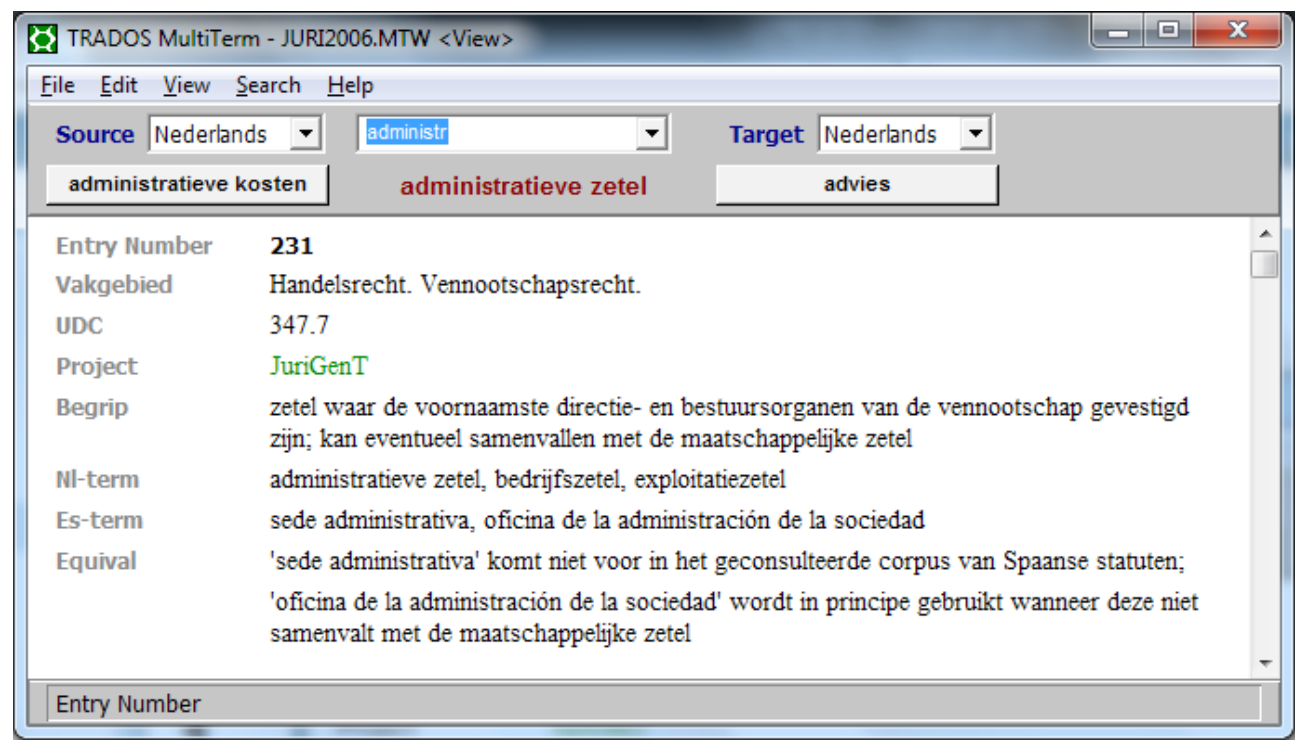

Figura 5. JuriGenT: 'administratieve zetel'/'sede administrativa’ (hoja de traducción)

Teniendo en cuenta, además, la importancia de las restricciones impuestas por los patrones colocacionales (Goźdź-Roszokowski 2013), hemos tenido que añadir en numerosas ocasiones la advertencia siguiente: "Los términos recogidos solo pueden considerarse como sinónimos hasta cierta altura; véanse las definiciones o contextos». En estos casos, ni los corpus ni las definiciones jurídicas nos permitían aclarar los matices semánticos. Para el neerlandés existen, por ejemplo, 'verrichting', 'activiteit', 'daad', 'handeling', 'taak', 'transactie', y sus equivalentes españoles 'actividad', 'acción', 'acto', 'operación' (figura 6).

Patricia VANDEN BULCKE y Carine DE GROOTE JuriGenT, un banco de datos jurídico neerlandés/ español diferente 


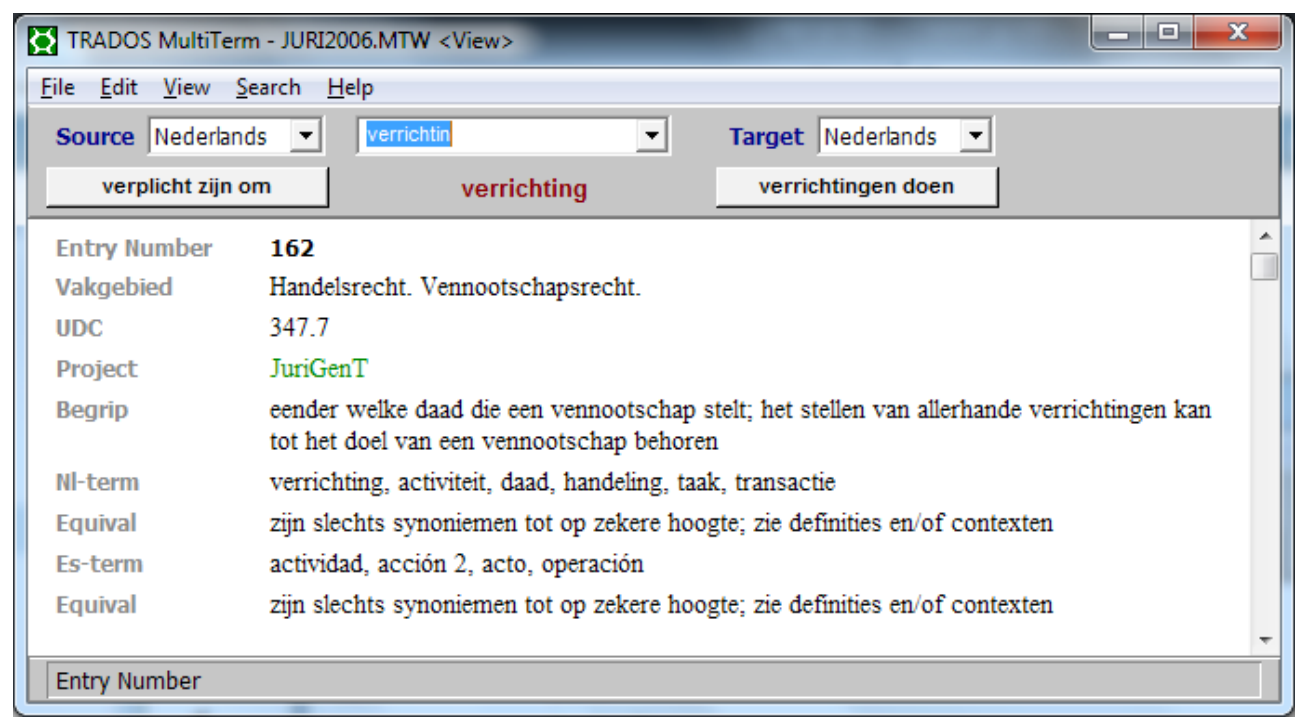

Figura 6. JuriGenT: 'verrichting'/'actividad' (hoja de traducción)

\subsection{Selección de unidades más amplia que en los diccionarios jurídicos tradicionales}

Este tema ha sido tratado ampliamente en Vanden Bulcke y De Groote (2011), por lo que aquí solo presentaremos un resumen de las conclusiones. La selección de las unidades de JuriGenT se ha hecho manualmente, sobre la base de una lista de frecuencia de las palabras de los corpus. Sin embargo, el criterio de selección no ha sido la frecuencia, sino la utilidad para el usuario: términos que aparecen una sola vez en uno de los corpus pueden figurar en JuriGenT si son relevantes para el traductor, por ejemplo porque no figuran en los diccionarios. Es el caso de los términos 'sociedad gestora de carteras' y 'beëindiging van de opdracht' (cese de las funciones).

En JuriGenT se distinguen tres tipos de unidades: las unidades terminológicas (UT), las unidades fraseológicas (UF) y las unidades de enlace (UE). Las primeras son las que tienen un claro contenido jurídico y suelen aparecer en los diccionarios jurídicos monolingües o bilingües, tanto términos simples como complejos o sintagmáticos: 'reserva'/'reserve', 'capital suscrito'/'geplaatst kapitaal', 'acción con derecho al dividendo anual mínimo'/'aandeel met een preferent dividendrecht en liquidatievoorrecht'. Sin embargo, nuestra experiencia docente y como traductoras jurídicas nos enseña que a menudo son las unidades situadas en la intersección entre la lengua jurídica y la lengua común las que plantean dificultades de traducción:

Patricia VANDEN BULCKE y Carine DE GROOTE JuriGenT, un banco de datos jurídico neerlandés/ español diferente 
'actividad' ('verrichting' al lado de 'activiteit'), 'supuesto' ('geval' y no 'veronderstelling'), 'advies' ('servicios de asesoramiento' mejor que 'asesoramiento'), etcétera. Por eso se han recogido como UT unidades de este ámbito límite: 'administración'/'bestuur', 'párrafo'/'alinea', 'urgencia'/'hoogdringendheid'... Nos parece «que forman parte de la jerga jurídica y de automatismos que resultan evidentes para el jurista, pero no para el traductor» (Vanden Bulcke y De Groote 2011, 89) y que, por lo tanto, deben incluirse.

Las unidades fraseológicas (UF) reciben en JuriGenT una definición pragmática: son las "colocaciones» - tal y como se definen en 4.2 de este artículo- para las que elaboramos una ficha como si fueran UT, aportando información sobre la fiabilidad, información gramatical, y también sinónimos y contextos; carecen de descripción conceptual. La selección de las UF se hace mediante introspección en función de nuestra experiencia como traductoras jurídicas. Predominan criterios de asimetría entre las dos lenguas tratadas. Hay UF basadas en criterios morfosintácticos, muy a menudo V + UT, y las hay basadas en criterios sintáctico-semánticos, con especial atención a la estructura argumental (número y jerarquía de argumentos, papel de agente o paciente...). Citamos como ejemplos de la primera categoría:

- realizar actividades (handelingen verrichten)

- dar comienzo a sus operaciones (verrichtingen vangen aan)

- participaties te gelde maken (venta de participaciones)

- tot de bevoegdheid behoren van (competer a)

Y de la segunda:

- de pleno derecho (van rechtswege)

- salvo acuerdo en contrario (behoudens andersluidend besluit)

- vrij zijn van achterstallige betalingen (hallarse al corriente en el pago de)

- voorafnemen tot vorming van de wettelijke reserve (deducir la dotación de la reserva legal)

- podrá acordar el traslado del domicilio social (de zetel kan verplaatst worden bij besluit van)

Patricia VANDEN BULCKE y Carine DE GROOTE JuriGenT, un banco de datos jurídico neerlandés/ español diferente 
Finalmente, están las unidades de enlace (UE): carecen de contenido especializado y tienen un gran potencial combinatorio con las UT del banco de datos. A diferencia de las UFn no contienen ninguna UT, sino que sirven de enlace entre UT. Suelen ser sintagmas cuyo núcleo puede ser un nombre, un elemento verbal, un adjetivo, un adverbio $u$ otros. Son relevantes para el traductor jurídico ya que contribuyen al «encaje textual» (cf. 4.2) de la traducción. Una pequeña investigación reseñada en Vanden Bulcke y De Groote (2011, 97-98) muestra que estas unidades no figuran en los diccionarios jurídicos monolingües, y solo parcialmente en el diccionario bilingüe DIC Oosterveld (1990). Remitimos para una lista provisional de las UE al artículo arriba mencionado. Véanse a título ilustrativo las figuras $7 a$ y $7 \mathrm{~b}$.

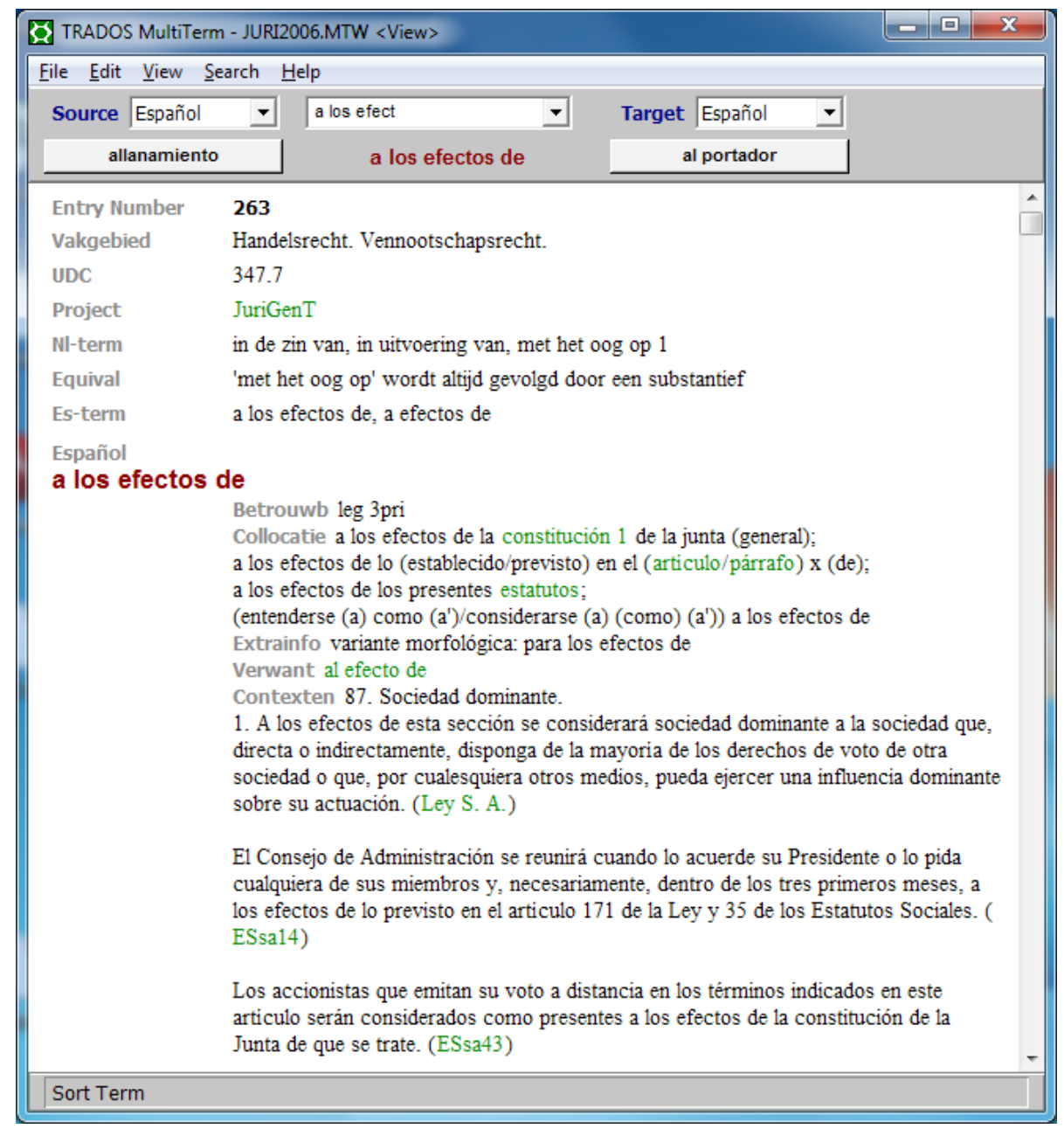

Figura 7a. JuriGenT: 'a los efectos de’ (hoja de traducción, hoja de lengua y hoja de texto)

Patricia VANDEN BULCKE y Carine DE GROOTE JuriGenT, un banco de datos jurídico neerlandés/ español diferente 


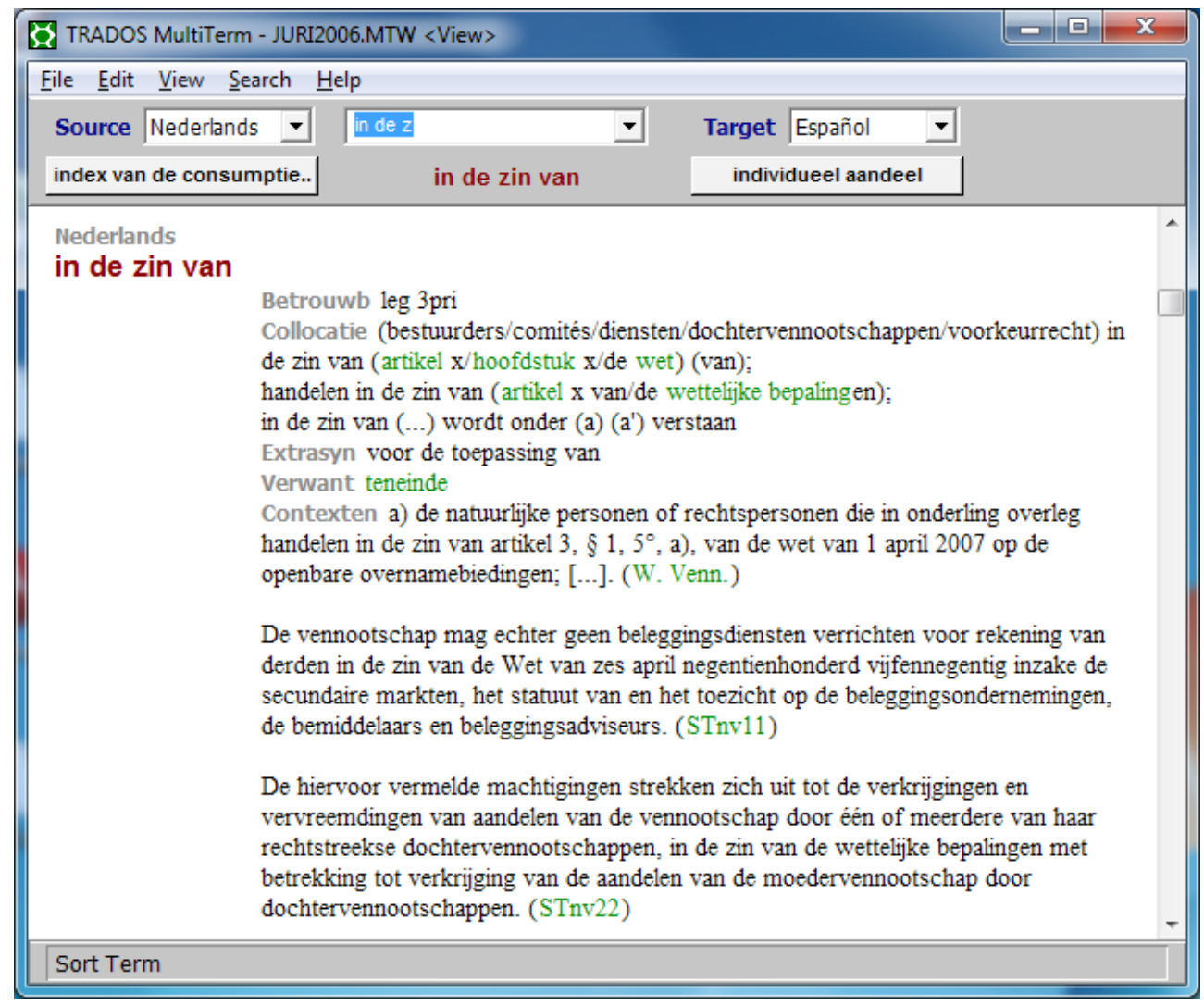

Figura 7b. JuriGenT: 'in de zin van' (hoja de lengua y hoja de texto)

\section{CONCLUSIONES}

Como bien dice el título, JuriGenT quiere ser un banco de datos jurídico bilingüe "diferente», sobre todo en el sentido de que se dirige específicamente al traductor jurídico. El propósito es que sea una herramienta que contribuya al «encaje textual» de la traducción, es decir, que ayude al traductor a producir un texto que se parezca lo más posible a un texto no traducido. El enfoque es, pues, contrastivo e influye tanto en el diseño de las fichas como en la descripción de los conceptos y la selección de las unidades recogidas.

En el diseño de las fichas destaca el llamado "campo de equivalencia", que permite registrar cualquier asimetría entre ambas lenguas. La descripción conceptual de las UT se basa en el intento de aplicar unos criterios jerárquicos (cf. los árboles nocionales con estructuras de coordinación, hiperonimia e hiponimia), eliminando el

Patricia VANDEN BULCKE y Carine DE GROOTE JuriGenT, un banco de datos jurídico neerlandés/ español diferente 
referente jurídico para facilitarle la comprensión al lego. Cabe mencionar, sin embargo, que cada unidad va acompañada, en lo posible, de una definición jurídica. En lo que concierne a la selección de las unidades, es preciso señalar a) que el banco de datos solo recoge unidades que se usan en la práctica (cf. los corpus), no unidades extraídas de monografías jurídicas y b) que se incluyen, además de las UT jurídicas, UT situadas en la intersección entre la lengua jurídica y la lengua común, y también colocaciones, algunas de ellas elaboradas como UF y UE. Son todas ellas unidades ignoradas por los diccionarios jurídicos tradicionales. Que conste, sin embargo, que ni siquiera «un banco de datos jurídico bilingüe diferente» consigue solucionar todos los problemas inherentes a una tarea tan compleja como la traducción jurídica. Aunque supone una ayuda valiosa, la «última palabra» la tiene el traductor «emancipado».

\section{REFERENCIAS BIBLIOGRÁFICAS}

ANDRADES MORENO, Arsenio. 2013. "La importancia de los binomios en la traducción jurídica». En Translating culture/Traduire la culture/Traducir la cultura, ed. por Emilio Ortega Arjonilla. Granada: Comares (Vol. 3), 401-413.

BHATIA, Vijay. 1994. "Cognitive Structuring in Legislative Provisions». En Language and the Law, ed. por John Gibbons. London: Longman, 136-155.

BIEL, Lucja. 2014. Lost in the Eurofog. The Textual Fit of Translated Law. Frankfurt am Main: Peter Lang.

CABRÉ, María Teresa. 1999. «La terminología. Representación y comunicación. Una teoría de base comunicativa y otros artículos». Barcelona: Institut Universitari de Lingüística Aplicada, Universitat Pompeu Fabra (Sèrie Monografies, 3).

CABRÉ, María Teresa. 2005. ¿Necesita un traductor saber y saber hacer terminología? Ponencia en el marco de la cátedra Karel V-Leerstoel. Universiteit Gent, Hogeschool Gent.

CABRÉ, María Teresa, Rosa Estopà, Judit Freixa, Mercè Lorente, Jaime Martí y Carles Tebé. 2003. "La enseñanza de la terminología en la traducción especializada: nuevas propuestas para viejos problemas». En Terminología y traducción: un bosquejo de su evolución, ed. por Natividad Gallardo San Salvador. Granada: Atrio, 117-129. http://www.upf.edu/pdi/iula/ merce.lorente/docums/comunic1.pdf.

CHESTERMAN, Andrew. 2004. «Hypotheses about Translation Universals». En Claims, Changes and Challenges in Translation Studies. Selected Contributions from the EST Congress (Copenhague 2001), ed. por Gyde Hansen, Kirsten Malmkjaer y Daniel Gile. Amsterdam: Benjamins, 1-13.

CHROMÀ, Marta. 2011. "Synonymy and Polysemy in Legal Terminology and their Applications to Bilingual and Bijural Translation». En Legal Terminology: Approaches and Applications. A special Issue of Research in Language 9 (1): 31-50.

ENGBERG, Jan. 2013. «Why Translators Are Not Lawyers. On Differences and Similarities of Interest and Knowledge». En Translating the Law. Theoretical and Methodological Issues, ed. por Icíar Alonso Araguás, Jesús Baigorri Jalón y Helen J. L. Campbell. Granada: Comares, 23-33.

Patricia VANDEN BULCKE y Carine DE GROOTE JuriGenT, un banco de datos jurídico neerlandés/ español diferente vol. 2-2, December 2016, 15-38

elSSN: 2444-1961

(c) Ediciones Universidad de Salamanca - CC BY-NC-ND 
EUROLECT OBSERVATORY. Interlingual and Intralingual Analysis of Legal Varieties in the EU Setting. http://www.unint. eu/it/ricerca/gruppi-di-ricerca/18-alta-formazione-it/407-osservatorioeuroletto.html

GoźDŹ-ROszOKOWSKI, Stanislaw. 2013. «Exploring Near-synonymous Terms in Legal Language. A Corpus-based Phraseological Perspective». En Research Models and Methods in Legal Translation, ed. por Lucja Biel y Jan Engberg. Linguistica Antverpiensia New Series 12: 94109. https://lans.ua.ac.be/index.php/LANS-TTS/issue/current

JURIGENT. (s.f.): Banco de datos jurídico bilingüe español/neerlandés en elaboración, Vanden Bulcke Patricia y Carine De Groote. Universiteit Gent, Vakgroep Vertalen, Tolken en Communicatie.

DE Groot, Gerard-René. 1987. «Het Vertalen van Juridische Teksten». En Recht en Vertalen, ed. por Jeppe Balkema y Gerard-René De Groot. Deventer: Kluwer.

DE Groot, Gerard-René. 1988. «Vertaalproblemen». En Een Inleiding tot het Rechtsvergelijkende Onderzoek», ed. por Dimitra Kokkini-latridou. Deventer: Kluwer, 94-103.

DE Groot, Gerard-René. 1996. Het Vertalen van Juridische Informatie. Deventer: Kluwer.

GEMAR, Jean-Claude. 1988. «La traduction juridique: art ou technique d'interprétation». Meta 2 (33): 305-319.

GEMAR, Jean-Claude. 2002. «Le plus et le moins-disant culturel du texte juridique. Langue, culture et equivalence». Meta 2 (47): 163-176.

KISCH, Isaac. 1973. «Droit comparé et terminologie juridique». En Inchieste di Diritto Comparato 2: Buts et Méthodes du Droit Comparé, ed. por Mario Rotondi. Padova/New York: Cedam/ Oceana.

LORENTE CASAFONT, Mercè. 2002. "Terminología y fraseología especializada: del léxico a la sintaxis». En Panorama actual de la terminología, ed. por Manuel Fernando Pérez Lagos y Gloria Guerrero. Granada: Comares, 159-180.

MARTIN, Willy. 2006. "Kleine talen maken het verschil: het vertaalwoordenboekenbeleid van de Nederlandse en de Vlaamse overheid in het laatste decennium». En Over woordenboeken, ed. por Willy Martin. Utrecht: Het Spectrum, 55-59.

MAYORAL ASEnSIO, Roberto. 2002. «¿Cómo se hace la traducción jurídica?». Puentes. Hacia nuevas investigaciones en la mediación intercultural 2: 9-14.

MAYORAL ASENSIO, Roberto. 2003. Translating Official Documents. Manchester: St. Jerome.

NORD, Christiane. 1997. «Defining Translation Functions. The Translation Brief as a Guideline for the Trainee Translator». Ilha do Desterro 33: 25-38.

ŠARČEVIĆ, Susan. 1991. «Bilingual and Multilingual Legal Dictionaries: New Standards for the Future». Meta 4 (36): 615-626.

ŠARČEVIĆ, Susan. 1997. New Approach to Legal Translation. The Hague: Kluwer.

ŠARČEVIĆ, Susan. 2000. «Legal Translation and Translation Theory: a Receiver-oriented Approach». Fecha de acceso 19 de enero de 2015. http://www.tradulex.com/Actes2000/ sarcevic.pdf.

TemmeRmAN, Rita. 2000. Towards New Ways of Terminology Description. The Sociocognitive Approach. Amsterdam/Philadelphia: John Benjamins.

THIRY, Bernard. 1997. «Bilingüismo y bijuridismo: problemas de equivalencia». En Introducción a la traducción jurídica y jurada (francés-español), ed. por Pedro San Ginés Aguilar y Emilio Ortega Arjonilla. Granada: Editorial Comares, 23-40.

Patricia VANDEN BULCKE y Carine DE GROOTE JuriGenT, un banco de datos jurídico neerlandés/ español diferente 
THIRY, Bernard. 2000. «Equivalence bilingue en traduction et terminologie juridiques. Qu'est-ce que traduire en droit ?». En La traduction juridique, histoire, théorie(s) et pratique, ed. por Hanne Lee-Jahnke. Genève : Éditions de l'Université de Genève, 285-307.

THIRY, Bernard. 2009. Terminología y derecho. La responsabilidad civil extracontractual. Contribución a su tratamiento terminográfico y a la teoría de la terminología. Granada: Editorial Atrio.

VANDEN BULCKE, Patricia. 1993. «Aspecten van de juridische vertaalproblematiek: Verantwoord 'vertalen' bij gebrek aan equivalente begrippen. Enkele Voorbeelden Spaans-Nederlands». Linguistica Antverpiensia 27: 225-239.

VANDEN BULCKE, Patricia. 1995. «Het begrip 'equivalentie' in juridische terminografie: Kritische benadering aan de hand van een casus Spaans-Nederlands». ITL-Review of Applied Linguistics 107-108: 79-88.

VANDEN BULCKE, Patricia. 1997. «De specificiteit van het juridisch discours en de dilemma's van de juridische vertaler: een inleiding». ITL-Review of Applied Linguistics 117-118: 51-78.

VANDEN BULCKE, Patricia. 2009. «¿Cómo tratar las asimetrías conceptuales en diccionarios o en bancos de datos jurídicos bilingües?». En El hispanismo omnipresente, homenaje a Robert Verdonk, ed. por Rita De Maeseneer et al. Antwerpen: University Press Antwerp, 309-318.

VANDEN BULCKE, Patricia. 2011. "JuriGenT, tweetalige juridische databank ontworpen voor de vertaler: knelpunten en oplossingen». En TiNT-dag 2010. Reeks Terminologie in het Nederlandse Taalgebied, ed. por Els Ruijsendaal y Cornelia Wermuth. Gent: Academia Press, 13-30.

VANDEN BULCKE, Patricia. 2013. «Dealing with Deontic Modality in a Termbase: the Case of Dutch and Spanish Legal Language». En Research Models and Methods in Legal Translation, ed. por Lucja Biel y Jan Engberg. Linguistica Antverpiensia New Series 12: 12-32. https://lans. ua.ac.be/index.php/LANS-TTS/issue/current.

VANDEN BULCKE, Patricia y Carine de Groote. 2011. "La base de datos terminológica jurídica bilingüe: O sobre la necesidad de la inclusión de unidades de enlace». ITL-Review of Applied Linguistics 162: 84-110.

VANDEN BULCKE, Patricia y Armand Héroguel. 2011. "Quality Issues in the Field of Legal Translation». En Perspectives on Translation Quality. Serie Text, Translation, Computational Processing, ed. por Ilse Depraetere. Berlin: De Gruyter Mouton, 211-248.

\section{Abreviaturas}

DIC Boutmans

BOUTMANS, Eddy. 2005. Juridisch lexicon Spaans-Nederlands met tal van politieke, diplomatieke en institutionele termen uit Spanje en Latijns-America. Con CD-ROM.

DIC Oosterveld

OOSTERVELD-EGAS REPARAZ, María Consuelo y Johanna VUYK-BOSDRIESZ. 1990. Juridisch woordenboek Nederlands-Spaans, met register Spaans-Nederlands. Apeldoorn/ Antwerpen: Maklu Uitgevers, 's-Gravenhage: T.M.C. Asser Instituut.

DIC Ribó Durán 2005

RIBÓ DURÁN, Luis. 2005. Diccionario de derecho. Barcelona: Editorial Bosch.

Patricia VANDEN BULCKE y Carine DE GROOTE JuriGenT, un banco de datos jurídico neerlandés/ español diferente 


\section{EUR-LEX}

HTTP://WWW.EUR-LEX.EUROPA.EU/NL/INDEX.HTM

IATE

INTER Active Terminology for Europe

HTTP://IATE.EUROPA.EU

Patricia VANDEN BULCKE y Carine DE GROOTE JuriGenT, un banco de datos jurídico neerlandés/ español diferente
CLINA

vol. 2-2, December 2016, 15-38 elSSN: 2444-1961

(C) Ediciones Universidad de Salamanca - CC BY-NC-ND 